\title{
Partial Discharge and Internet of Things: A Switchgear Cell Maintenance Application using Microclimate Sensors
}

\author{
Radu Fechet ${ }^{1}$, Adrian I. Petrariu ${ }^{2, *}$ and Adrian Graur ${ }^{3}$ \\ Affiliation 1; radu.fechet@usm.ro \\ Affiliation 2; apetrariu@usm.ro \\ 2 Affiliation 3; adrian.graur@usv.ro \\ * Correspondence: apetrariu@usm.ro
}

\begin{abstract}
This paper proposes a solution for the development of microclimate monitoring for Low Voltage / High Voltage switchgear using the PRTG Internet of Things (IoT) platform. This IoT-based real time monitoring system can enable predictive maintenance to reduce the risk of electrical station malfunctions due to unfavorable environmental conditions. The combination of humidity and dust can lead to unplanned electrical discharges along the isolators inside a low or medium voltage electric table. If no predictive measures are taken, the situation may deteriorate and lead to significant damage inside and outside the switchgear cell. Thus, the mentioned situation can lead to unprogrammed maintenance interventions that can conduct to the change of the entire affected switchgear cell. Using a low-cost and efficient system, the climate conditions inside and outside the switchgear is monitored and transmitted remotely to a monitoring center. From the results obtained using a 365-day time interval, we can conclude that the proposed system is integrated successfully in the switchgear maintaining process, having as result the reducing of maintenance costs.
\end{abstract}

Keywords: electrical grid, switchgear, preventive maintenance, IoT, partial discharge

\section{Introduction}

Preventive maintenance and predictive maintenance are designed to reduce the amount of reactivity to failures and to increase the reliability of assets, both being a form of a scheduled maintenance. As is presented in Figure 1, there are four types of maintenance depending on the execution time: proactive (the shortest and the cheapest), preventive, predictive and reactive (the longest and the most expensive). These estimations from Figure 1 are made by a real-life experience of the authors in the telecommunication domain and can be extended for all domains that involves using equipment's and hardware infrastructure.

Preventive maintenance is based on work orders that are well scheduled before maintenance is performed. This type of maintenance can be compared to an annual or biannual physical check, to prevent future breakdowns or emergency maintenance issues and extend the functional life of assets. One of the main challenges with preventive maintenance is balancing the cost with returns. Thus, in this point an experienced maintenance manager is needed to take smart decisions regarding which devices require preventive maintenance and how frequently is needed.

Predictive maintenance is scheduled based on the actual equipment condition or asset conditions and reduces material costs and labor. The main difference between the two is that predictive maintenance is scheduled as needed, based on asset conditions while preventive maintenance is scheduled at fixed intervals, in a regular way. Implementing a predictive maintenance program requires resources as money and training. These costs are often acceptable to companies that have already successfully implemented a predictive maintenance program. Nowadays, many companies are on the right part of the potential-to-failure curve, doing reactive work, meanwhile some companies are doing predictive maintenance to reach the proactive maintenance. According to the Figure 1, the 
reactive domain has negative effects as increasing the maintenance costs, causing the unscheduled downtimes, forcing the company to perform unplanned work, and spent higher costs to shipping spare parts.

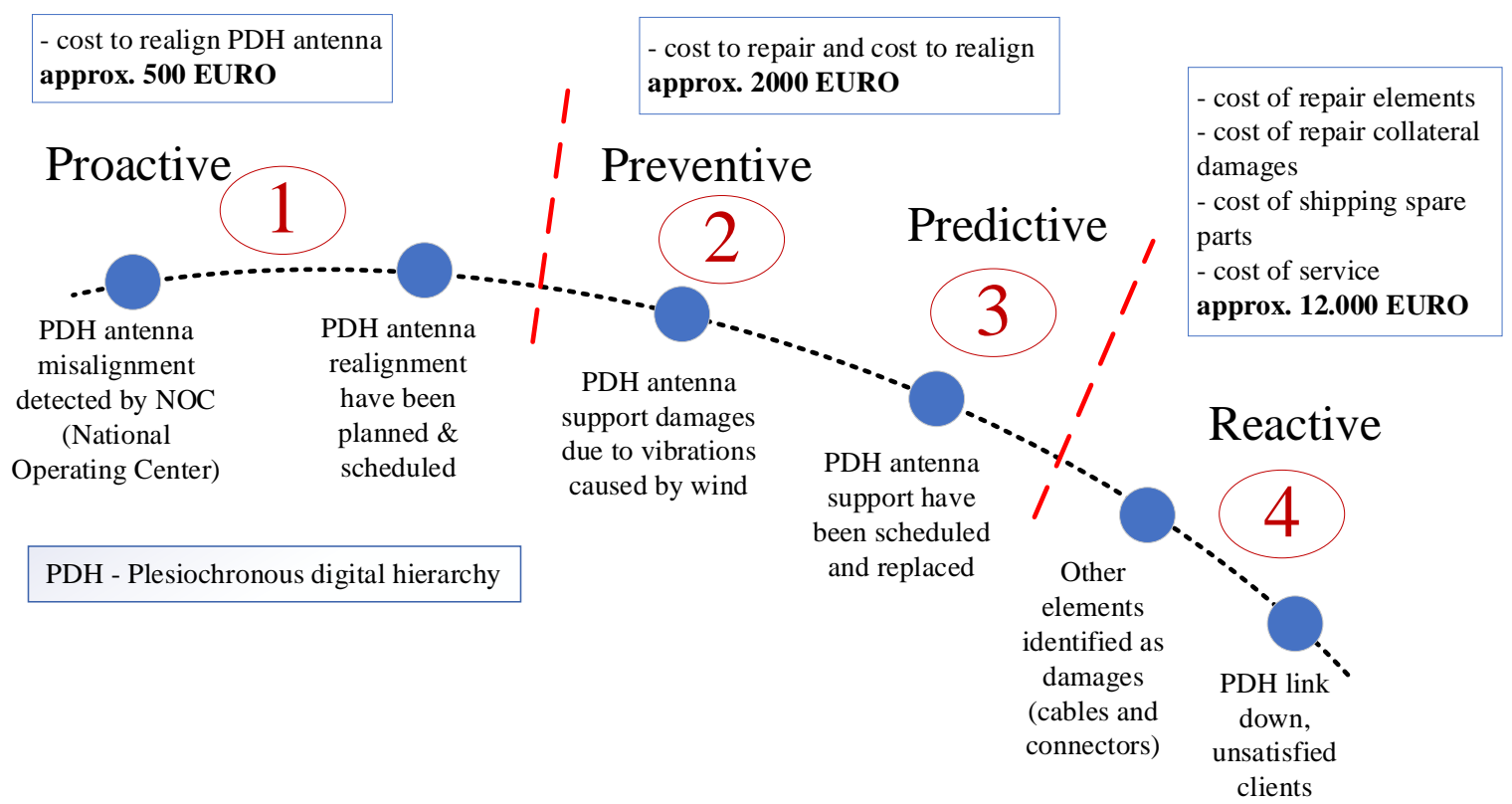

Figure 1. Example of maintenance costs in telecommunications and potential-to-failure curve

Generally, for implementing predictive maintenance some of the main components are necessary such as: early fault detection, fault detection, time to failure prediction, data collection and preprocessing, resource optimization and maintenance scheduling. Predictive maintenance has also been one of the driving forces for improving productivity [1].

In evaluating equipment condition, predictive maintenance use non-destructive testing technologies such as: sound level measurements, vibration, infrared, acoustic (partial discharge and airborne ultrasonic) or oil analysis, corona detection, inside camera monitoring and other specific online tests. In most of the applications, the site measurements are supported by wireless sensor networks (WSN's) to reduce the wiring cost and for easy configuration of the measurement setup [2].

In collaborative process automation systems (CPAS) there are performed two types of measurements: one on the actual equipment and one involving process performance measured with help of other devices. Thus, the equipment maintenance is triggered. Predictive maintenance can evaluate the condition of equipment by performing continuous (online) or periodic (offline) equipment conditions monitoring. Remote monitoring by collecting data continuously can help preventive care and can also diagnose failures earlier using Internet of Things (IoT) technologies. When the Low Voltage or Medium Voltage switchgear cells (LV/MV) are involved for example, a permanent monitoring of the environmental conditions is required. These aspects are needed to prevent electrical failures due to partial discharges (PD) and extend the assets lifetime. These environmental conditions may be temperature, humidity, dust, and ozone, aside from the PD sensor. Predicting the remaining useful life of an asset using supervised machine learning (ML) is the most common technique in predictive maintenance as is mentioned by the scientific researchers in [3] and [4].

In this paper we will present the concept design, development and implementation of a low-cost, open-source monitoring system based on the Internet of Things (IoT) architecture. The proposed system implies minimum system requirements and we attempted to use low-cost, low-power, reliable and readily available components to achieve the desired functions of a monitoring system. Analyzing the experiments performed using the proposed monitoring architecture, we show that one of the proposed systems works well by testing it with IoT specific modules and infrastructure. 


\section{Partial discharge (PD) in LV/MV switchgear cells}

Switchgear is an important element in an electrical grid that has control and protective role, respectively. Via the switchgear, it is possible to interrupt an electrical circuit of a factory/production line or for powering a town/village, e.g., to modify/upgrade parts of the schematic circuit or to prevent further damages of the electric grid after a fault usually located downstream the grid. There are many different types of switchgears, one of them being presented in Figure 2, manufactured by ElectroAlfa, a profile company from Romania. The main parts of a switchgear are listed in the Figure 2.

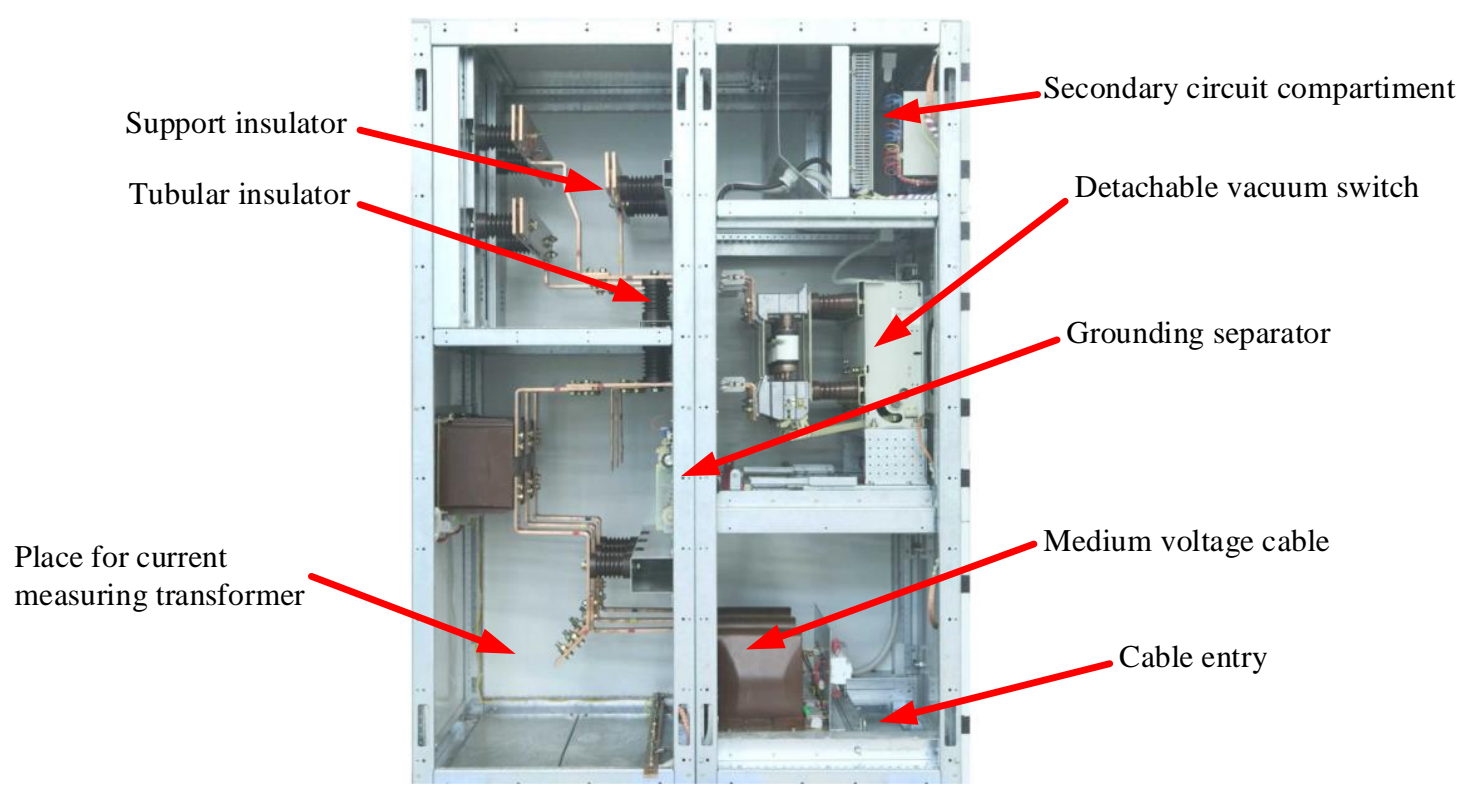

Figure 2. Example of MV switchgear [5]

In electrical domain, partial discharge (PD) is an electrical discharge between two electrical components that does not completely bridge the space between two conducting electrodes which are at different electrical potential in medium and high voltage equipment's. The discharge may occur in a gas filled chamber, a solid insulating material, a gas bubble, a liquid insulator, or around an electrode merged in a gas tank [6]. When partial discharge occurs in a gas environment, it is usually known as a corona [2], [7].

According to IEC 60270 standard, PD is generally divided into two major sub-groups, named internal and external PD, depending on their occurrence [8]. Partial discharge (PD) occurs in gas-filled cavities or defects in the high voltage insulation and can have the following possible causes: proper manufacture failure, equipment installation, excessive use of the equipment while in service, in-service damage due to external environmental factors, ageing and deterioration, particle, and dust contamination [9]. PD within solid insulation system is not visible, can corrode solid insulation and eventually lead to insulation breakdown. PD can leave visible marks such as carbon tracks, discolored lines, and odors (metallic, burning or ozone). Also, a PD can cause damages that can be chemical, thermal, mechanical and emits gasses and different forms of energy such as electromagnetic or acoustic.

Due to ozone emission from PD events and because ozone is an oxidizing agent, PD is a main cause of long-term degradation and eventual failure of electrical insulation, ultimately leading to complete failure. As a result, its measurement is a standard procedure in the factory testing setup of many types of high voltage equipment. Thus, PD activity can be tested or monitored for the in-service equipment to warn against pending insulation failure.

In Figure 3 are depicted some of the PD energy emission types which we intend to measure in this paper using IoT infrastructure. To identify environmental conditions that facilitates the PD occurrence, continuous monitoring of gases and of electromagnetic or 
acoustic waves is crucial [10,11]. If a PD starts, the damages of the electrical equipment will exponentially increase and could cause safety issues, the process being irreversible.

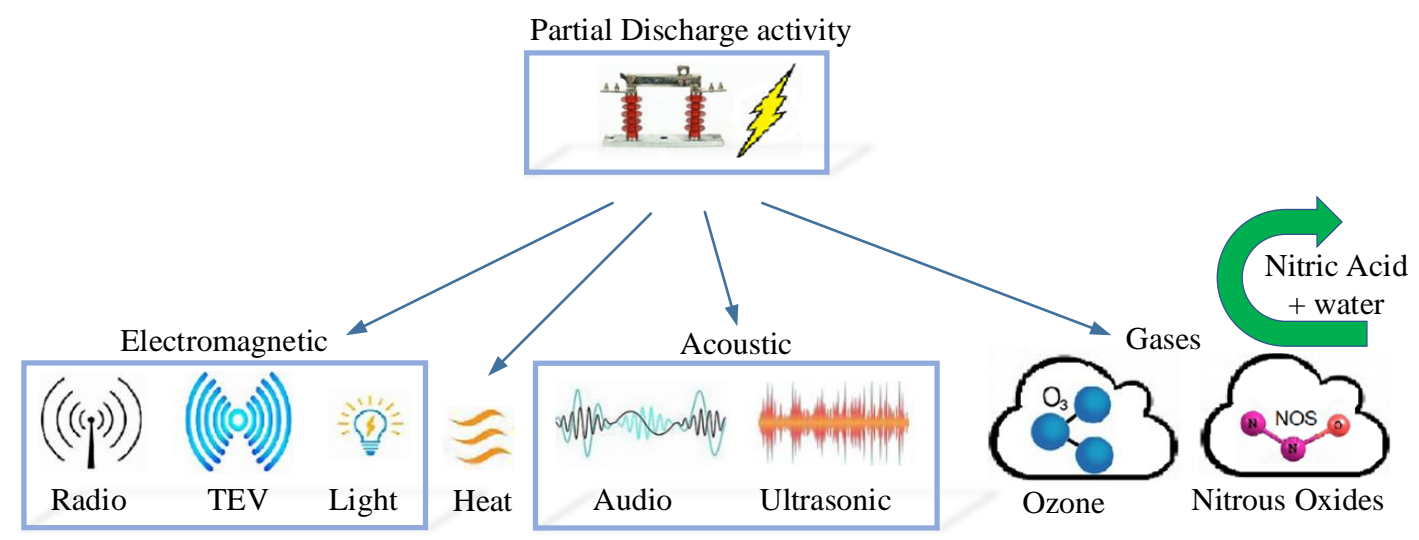

Figure 3. Partial discharge energy emissions

\subsection{PD (Partial Discharge) Detection}

For non-intrusive testing, the most practical techniques are based on the ultrasonic and radio frequency (RF) waves detection as part of the emissions in the electromagnetic spectrum (see Figure 3).

The acoustic method for PD detection is a very attractive alternative because acoustic signals from a PD source are immune to the electromagnetic noises. Because the switchgear is grounded, acoustic sensors can be positioned in a safe way in any point of the switchgear wall to detect the acoustic emission of any PD that can occur. Acoustic and ultrasonic sensors with a frequency band range from $10 \mathrm{kHz}$ to $300 \mathrm{kHz}$, like condenser microphones, sound-resonance sensors, accelerometers, or piezoelectric transducers, could detect an acoustic signal from mechanical vibration of any PD. When partial discharge is approaching to equipment failure, the emissions frequency decreases sometimes to the audible range. Another strong point of the acoustic method is that the acoustic signal has dependence upon the geometry of the DUT (Device Under Test). Thus, acoustic method is very used in localization of PD source inside switchgear because of its immunity against electromagnetic noise. There are also two problems: the acoustic wave propagation and the required sensitivity of the acoustic signal (very low intensity), so, to detect PD, sensors must be sensitive to small changes in amplitude of the measured signal. Using the airborne ultrasonic microphone is a sensitive method of air path of PD detection. Usually, PD detection devices using ultrasonic sensors convert emissions into audible signals so it can hear the PD activity.

PD activity which causes Transient Earth Voltages (TEV) were first discovered by Dr John Reeves in the 1970s at EA Technology. Dr. John Reeves concluded that PD activity and TEV signals have a direct dependence and could be an indication of the status of switchgear insulation. If the switchgear is in service, PD activity may be detected by placing sensors on the outside of the switchgear.

Ultra-High Frequency (UHF) PD detection was mostly oriented to gas insulation substation (GIS) at the beginning. After years of research, the ultra-high frequency partial discharge detection can be used also in non-gas insulation electrical power devices such as transformer, cables, switchgears, etc.

PD detection using optical sensors have dependence on different factors such as temperature, pressure, $\mathrm{PD}$ intensity and insulation material. Mainly there are two techniques of optical PD detection, surface detection and the opto-acoustic measurement. The main advantage of the last method is high sensitivity and immunity from electromagnetic interferences. 
Switchgear PD activity can be identified and saved using several methods, all depending on the sensor technologies used. Below it is a brief presentation.

- Ultrasonic microphone with a center frequency of $40 \mathrm{kHz}$. Measuring devices are available to convert PD ultrasonic emissions into audible signals, so it can detect changes in PD activity. Data from ultrasonic sensors can also be displayed and recorded locally as numerical decibel values.

- Transient Earth Voltage (TEV) sensor or coupling capacitor, from $3 \mathrm{MHz}$ to $100 \mathrm{MHz}$. In this case, TEV signals are measured, displayed, and locally recorded using the appropriate instrument. The sensitivity is not likely to be sufficient to detect issues within solid dielectric cable systems.

- Ultra-High Frequency Sensor (UHF), with a detection bandwidth between $300 \mathrm{MHz}$ and $1.5 \mathrm{GHz}$. UHF radio emissions are also measured, displayed, and locally saved on a dedicated device. More sensitive measurements, particularly at higher voltages, can be achieved using built-in UHF antennas or external antennas mounted on insulating spacers in the surrounding metalwork.

- High Frequency Current Transformer (HFCT) with a bandwidth between $500 \mathrm{kHz}$ to $50 \mathrm{MHz}$. This method is ideal for detecting and determining the severity of the PD by burst interval measurement. The closer the bursts get to "zero voltage crossing" the more severe and critical the PD fault is.

- Acoustic Contact Sensor with detection bandwidth between $20 \mathrm{kHz}$ to $300 \mathrm{kHz}$.

- Phase resolved analysis system, which compares pulse timing to AC frequency.

- New studies present another method to detect $\mathrm{PD}$ by measuring the $\mathrm{O}_{3}$ and $\mathrm{NO}_{x}$ gases $[12,13]$, which in our opinion would be a cheaper solution compared to the abovementioned solutions.

Theory reported in [14] and [15] explains why corona and surface discharges have higher frequency spectra compared to internal PD. Therefore, frequency spectra of the surface discharges are higher than the frequency spectra of the internal PDs.

\subsection{PD (Partial Discharge) Monitoring}

To detect PD activity in switchgear we need a continuous monitoring of the cell. Thus, the PD activity must be recorded and the same sensor technologies as Partial Discharge measurements are used for data acquisition. The sensors will monitor:

- Changes in Partial Discharge activity over time.

- Changes in Partial Discharge activity related to environmental conditions, including vibrations, temperature, and humidity.

To record an analyze changes in PD activity over a dedicated period time, mobile PD monitoring systems are typically used, and an example is the one from EA Technology, named UltraTEV Monitor (Figure 4).
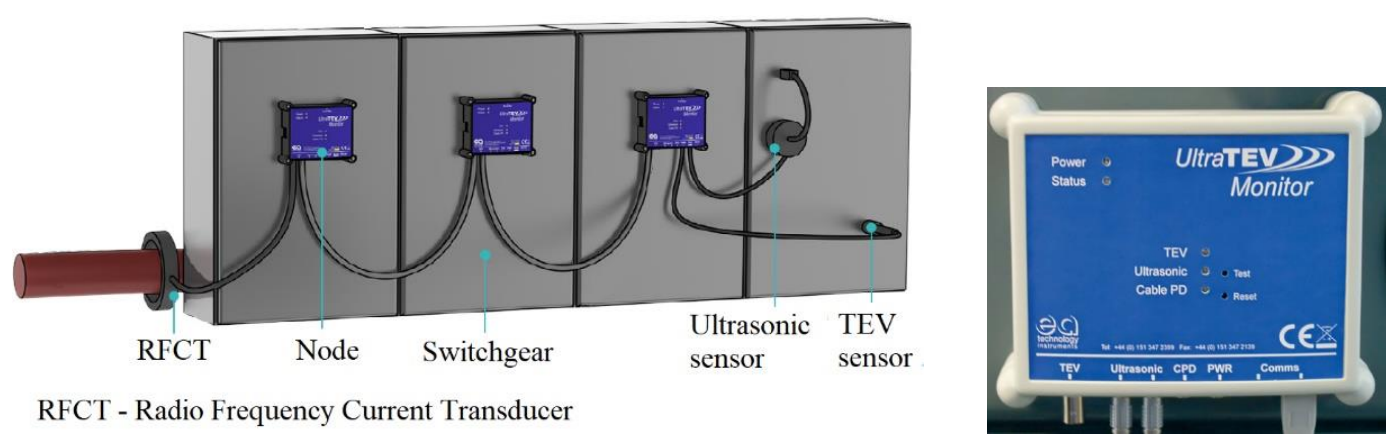

RFCT - Radio Frequency Current Transducer

Figure 4. System layout of an UltraTEV Monitor System and an UltraTEV node [16]

These portable devices include multiple sensors which can be mounted on a various type of assets and can be linked to several central server units for data storage and remote access. Thus, PD data can be collected and analyzed in online or offline mode. This type 
of monitoring system is often used to provide a status regard the health of the assets which have history events and/or where ongoing reliability is a critical issue.

\subsection{Ozone measurements in PD (Partial Discharge)}

Ozone $\left(\mathrm{O}_{3}\right)$ is a highly reactive gas consisting of three oxygen atoms with an unstable temperature dependent connection between atoms. Its half-life in the air is of 3 days at $+20^{\circ} \mathrm{C}$ and can go to 3 months at $-50^{\circ} \mathrm{C}$. Inside buildings, the indoor $\mathrm{O}_{3}$ half-life is usually less than 30 minutes and its typically value is between 5 to $20 \mathrm{ppb}$ (parts per billion). This gas can be a natural product in the stratosphere $\left(\mathrm{O}_{3}\right.$ layer being at $15 \mathrm{~km}$ to $45 \mathrm{~km}$ above sea level) or can be man-made in the troposphere (ground level).

The stratospheric $\mathrm{O}_{3}$ is formed naturally by interaction of sun's ultraviolet rays (UV) with oxygen $\left(\mathrm{O}_{2}\right)$. The $\mathrm{O}_{3}$ layer reduces the volume of harmful solar UV radiation on the Earth surface like a shield.

$\mathrm{O}_{3}$ at the ground level can be found also naturally in small concentrations of 20 to 35 ppb or because of human activity when is formed from photochemical reactions (UV) on polluted air with two major pollutants, nitrogen oxides $\left(\mathrm{NO}_{\mathrm{x}}\right)$ and volatile organic compounds (VOC). Nitrogen oxides can result primarily from combustion at high temperature from industrial boilers and furnaces, electrical powerplants and combustion motor vehicle. Within the last decade, higher ambient $\mathrm{O}_{3}$ concentrations have been observed in the presence of sunlight and heat in summer months, and in cold months, when temperatures are below or near freezing point and snow is on the ground. $\mathrm{O}_{3}$ contributes to the urban "smog" which manifests especially in the summertime and sometimes in mountain regions, and it is an important element in the measurement of the Air Quality Index (AQI).

When external PD occurs in electric LV/HV switchgear cells, the chemical reaction can produce $\mathrm{O}_{3}$, because electrical discharges usually have sufficient power to convert $\mathrm{O}_{2}$ from the air into $\mathrm{O}_{3}$. Following the mentioned, the switchgear could be a potential source of $\mathrm{O}_{3}$ and can be an indirect measurement parameter to prove the PD presence. Because $\mathrm{O}_{3}$ is a powerful oxidizing gas, its reaction with oxidizable materials such as polymeric or ferrous metal leads to their consumption and to decrease of $\mathrm{O}_{3}$ concentration. In the air, $\mathrm{O}_{3}$ reactions with other contaminants also consume $\mathrm{O}_{3}$ through oxidation and environmental conditions such as temperature and moisture can facilitate these reactions [17].

To compare the air in an LV/MV switchgear cell after PD, it is very important to analyze the initial values of $\mathrm{O}_{3}$ and $\mathrm{NO}_{x}$ in absence of the PD. It is important to note that different times of the same day have various background data for $\mathrm{O}_{3}$ and $\mathrm{NO}_{x}$. According to [13], some studies were performed regarding online monitoring of PD by measuring the air chemical structure. The experiments are conducted in different air humidity level conditions (30\% and $60 \% \mathrm{RH})$ at different gap distances $(15,20,25$ and $30 \mathrm{~mm})$. Their results showed that after a PD, the average value of air decomposition by-products is 5 to $10 \mathrm{ppb}$ higher in case of low humidity depending on gap distance as compared with high humidity conditions when constant voltage is applied $(4 \mathrm{kV})$. Finally, the study's conclusion was that PD could be easily detected in case of low humidity conditions, as compared with high humidity conditions. This gas analysis method could be an effective way to easily monitor PD.

The best solution is to use ultrasonic discharge counters in association with humidity and temperature sensors. New studies relate $\mathrm{O}_{3}$ sensors that could replace the ultrasonic discharge counter in some situations [17]. The number of this sensor devices could be: one ultrasonic discharge sensor because it is expensive and more than one humidity and temperature sensors, because they are cheap. Using a cheap integrated humidity and temperature sensor like DHT22 or DHT20 could be a very good solution. Another sensor like BME680 from BOSCH offers integrated multiple purpose sensors in the same chip, like temperature, humidity, ambient pressure, and gas. Dew point calculation using filtered and mediated data from the above sensors could provide good information regarding the possibility of the occurrence of PD conditions. Below is a color code for BOSCH's recommended air quality, colors that we intended to use in the software monitoring for the visual identification of the air quality. 


\begin{tabular}{|c|c|}
\hline IAQ Index & Air Quality \\
\hline $0-50$ & good $^{10}$ \\
\hline $51-100$ & average \\
\hline $101-150$ & little bad \\
\hline $151-200$ & bad \\
\hline $201-300$ & worse $^{2}$ \\
\hline $301-500$ & very bad \\
\hline
\end{tabular}

Figure 5. BME680 Indoor Air Quality (IAQ) and Color Coding according with the datasheet

\section{Proposed microclimate monitoring architecture for LV/MV switchgear cell stations}

To provide some data to analyze the PD discharge event in an LV/MV switchgear cell stations we use a hardware flowchart (Figure 6) and the architecture from Figure 7.

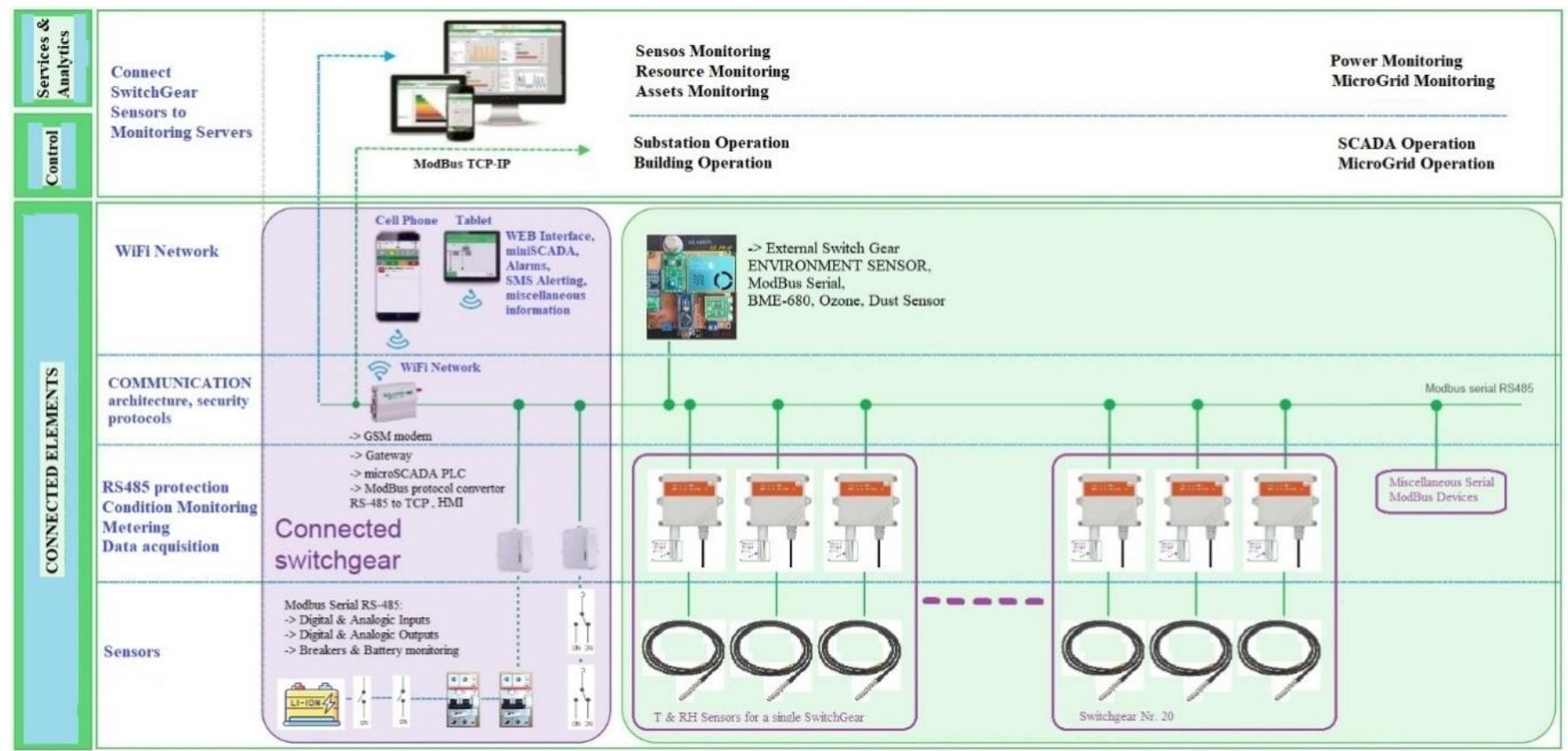

Figure 6. Hardware flowchart proposal for microclimate monitoring in LV/MV switchgear cells

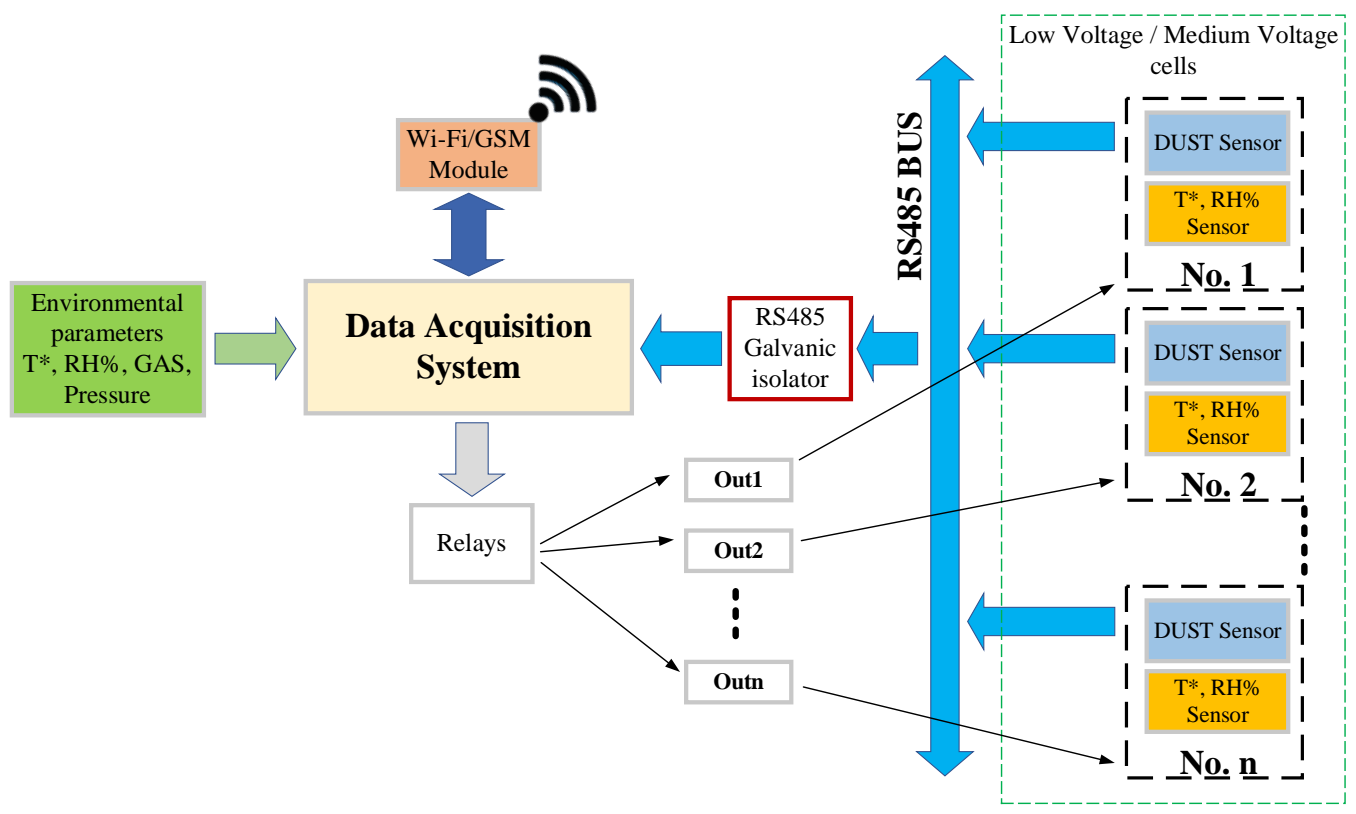

Figure 7. Base schematic proposal for microclimate monitoring in LV/MV cells 
Because the proposed devices will work in very harsh environmental conditions (excessive temperature, humidity, and dust values), the main components for this project must work with industrial or automotive performances. Therefore, our proposal for the hardware components is:

- Data Acquisition System (a dual band GSM router with Linux Embeded and RS485 interface).

- DC/DC power supply (from 48VDC - 230VDC to 12VDC) with a backup battery with minimum autonomy of $24 \mathrm{~h}$ in case of the main power supply loss. The lack of the power must be transmitted to the SysLog server and to a mobile terminal at the permanent maintenance service.

- Miscellaneous atmospheric and gas sensors with RS-485 interface for outside (room) and inside (LV/MV switchgear) measurements.

- GPIO's from Data Acquisition System to ensure relay commands for power control of the miscellaneous sensors.

Each LV/MV switchgear cell has integrated sensors for dust, temperature, and humidity. The sensors from the LV/MV switchgear cells transmit data using RS-485 BUS. Between the LV/MV switchgear cell stations and the Data Acquisition System is placed a galvanic isolator to protect the system from eventually faults that can occur with the communication lines. The role of the DAS (Data Acquisition System) is to provide enough data to be analyzed in the monitoring center. Some external sensors are mounted as well in the LV/MV cell station room to have a proper overview of the environment atmospheric values (temperature, humidity, gas, and air pressure). The room environmental parameters are important because they will provide functioning conditions of the switchgears and can predict eventually faults of the entire station. The communication with the monitoring center will be made through the GSM network, using GPRS (2G) or 3G/4G technologies, depending on the coverage from the area where LV/MV cells are in the field. GSM measurements need to be performed before installing the equipment, to determine if the network operators have the best coverage and the proper bandwidth for data transmission. For communication redundancy, a dual SIM GSM module modem is proposed. In case if some faults are detected, the monitoring center can transmit data to the LV/MV cell station and using some GPIO's from the DAS and some relays, the cell can be disconnected from the grid. The monitoring center architecture is listed in Figure 8. All the LV/MV switchgear stations from the field will transmit data and according with the Figure 8, the monitoring center will analyze the received data and will display using a GUI (graphical user interface) important information about the collected parameters.

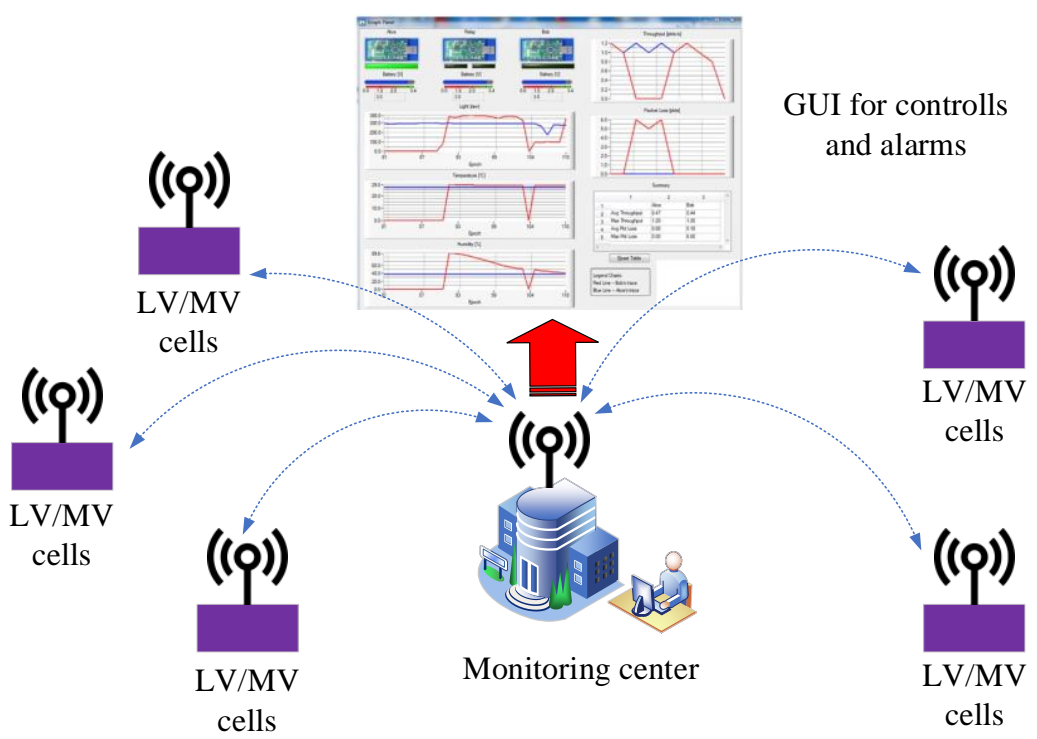

Figure 8. Communication flowchart proposal for microclimate monitoring in LV/MV switchgear 
In the next section it is briefly described data transmission between devices-server, devices-cloud platform or between IoT devices. For our scenario only two transmission protocols are used, named ModBus and MQTT (Message Queuing Telemetry Transport).

ModBus protocol technology in our proposed architecture is used for connecting the LV/MV switchgear sensors to the acquisition system. There are currently a lot of sensors on the market using the ModBus protocol and open-source software that includes a rich palette of development languages such as: C, Java, Python, Node.js and Go.

MQTT is a lightweight publish/subscribe messaging protocol that can be used for M2M IoT (Machine to Machine) communications [18]. The main characteristics of MQTT are low power consumption, small code size, minimized data packet size, and the possibility to distribute different packets to multiple applications almost at the same time. Any network-wired or wireless device that provides a bi-directional connection can support MQTT. This MQTT protocol was specifically designed for high latency networks or low bandwidth resource constrained devices.

The monitoring interface was designed to be available in two places: locally (SW microSCADA PLC) and remotely on more than two separate servers and using information security protocols.

\subsection{Local monitoring of LV/MV switchgear}

For local monitoring, a minimum SCADA will be created in DAS to observe the query of the sensors and to make a statistic for the local ModBus packages. A more likely SCADA system web page is made on the GSM router, where are related the LV/MV cells together with the sensors. This page must be low RAM memory consumption, to not affect the GSM router OS performances.

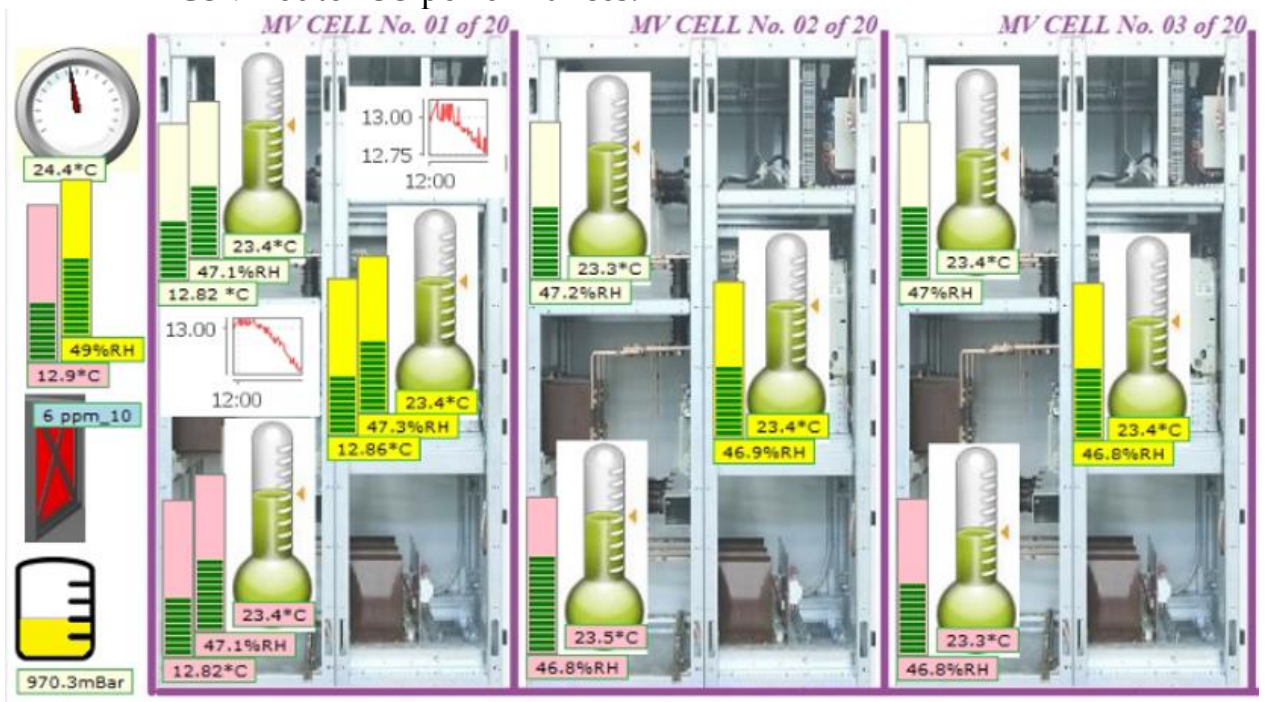

Figure 9. Example of locally microclimate monitoring using SCADA web page with sensors for 3 cells

Thus, with the obtained counters for serial ModBus packages, a local QoS (Quality of Service) statistic can be created that can be sent later to the server. The sizes obtained after interrogating the sensors should be stored locally for at least 7 days, which leads to the need for adequate sizing of RAM and circular buffers in the GSM router.

An example of local monitoring (local minimum SCADA) is presented below where the connection between the decrease of atmospheric pressure, the appearance of storms and the subsequent increase of the relative humidity in the atmosphere is observed. 


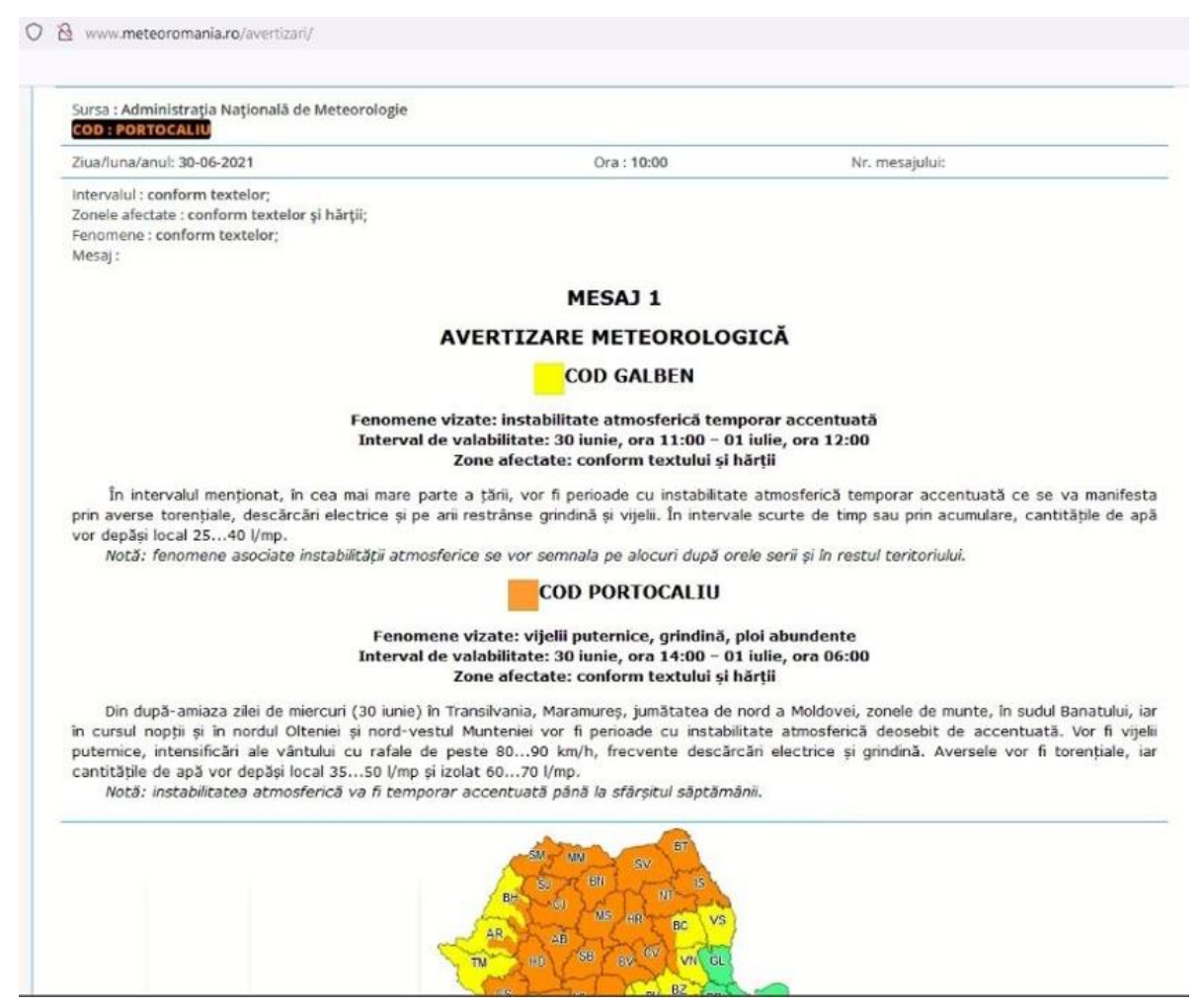

Figure 10. Example of Romanian weather warning, from 30-june-2021 to 01-july-2021

Increasing the air humidity leads to the increase of the soil humidity also. This aspect will have the effect of increasing the humidity in the cable compartments for the LV / MV cells located in the field, especially for those located in high altitude geographic areas (mountains for example). Increased humidity in the cable compartment can lead to Corona discharge phenomena, especially on the surface of the insulators in the cable compartment. Corona discharge or PD (Partial Discharge) lead to the release of gases including ozone $\left(\mathrm{O}_{3}\right)$ and nitrogen dioxide $\left(\mathrm{NO}_{2}\right) . \mathrm{NO}_{2}$ gas in the presence of the water vapors and oxygen $\left(\mathrm{O}_{2}\right)$ leads to the appearance of hydrochloric acid $\left(\mathrm{HNO}_{3}\right)$. Both ozone $\left(\mathrm{O}_{3}\right)$ and hydrochloric acid $\left(\mathrm{HNO}_{3}\right)$ are oxidizing elements that can irreparably destroy over time the insulation of cables and other insulating materials in the compartments of an LV / MV cell.

An example of this measurement is performed, and the results are depicted in Figure 11 and Figure 12, respectively.

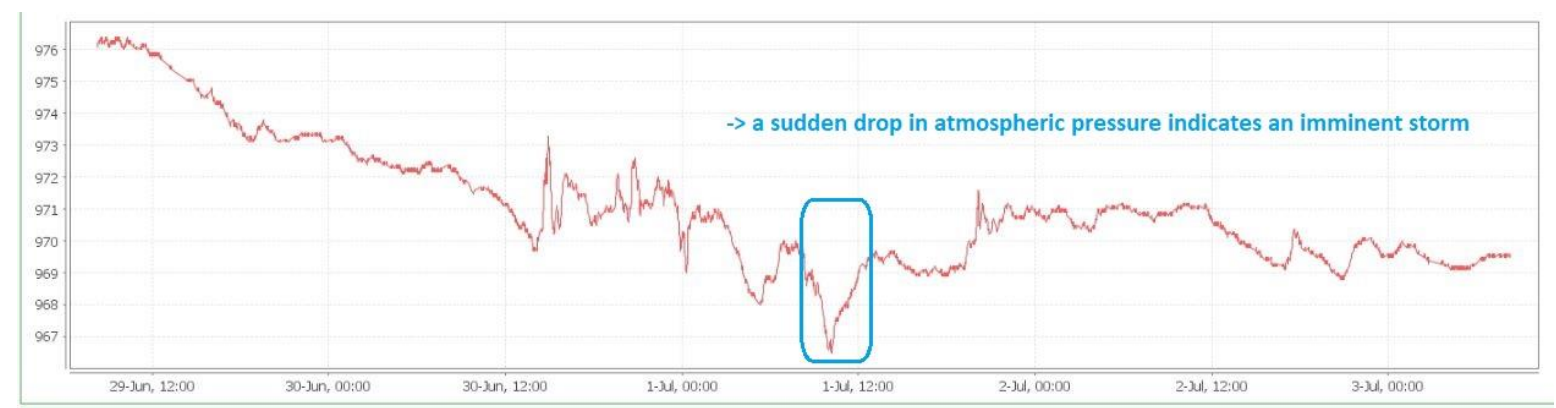

Figure 11. Local microclimate monitoring in LV/MV switchgear cells, indication of imminent storm: sudden drop in atmospheric pressure in 01-july-2021 before 12:00 PM 


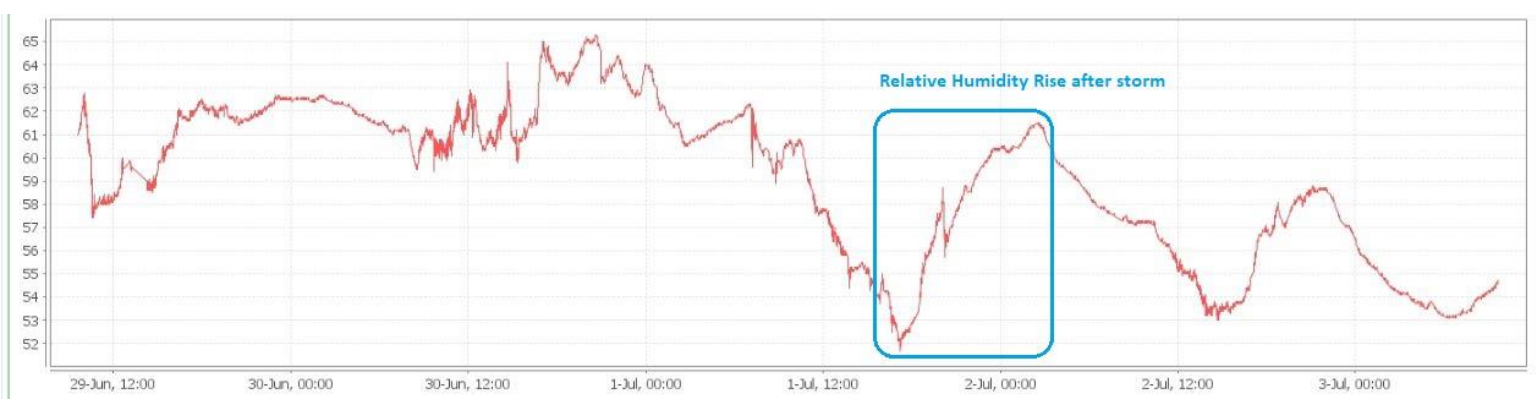

Figure 12. Local microclimate monitoring in LV/MV switchgear cells, increase in humidity after the storm of 01-july-2021

\subsection{Remote monitoring of LVIMV switchgear}

For the server side, the PRTG application (www.paessler.com) is currently being tested and some collected data are listed below. In the future the Zabbix application will be also tested (https://www.zabbix.com/) due to the advantages offered on the monitoring side for the MQTT protocol in the IoT plugin package in Zabbix Agent 2 [19].

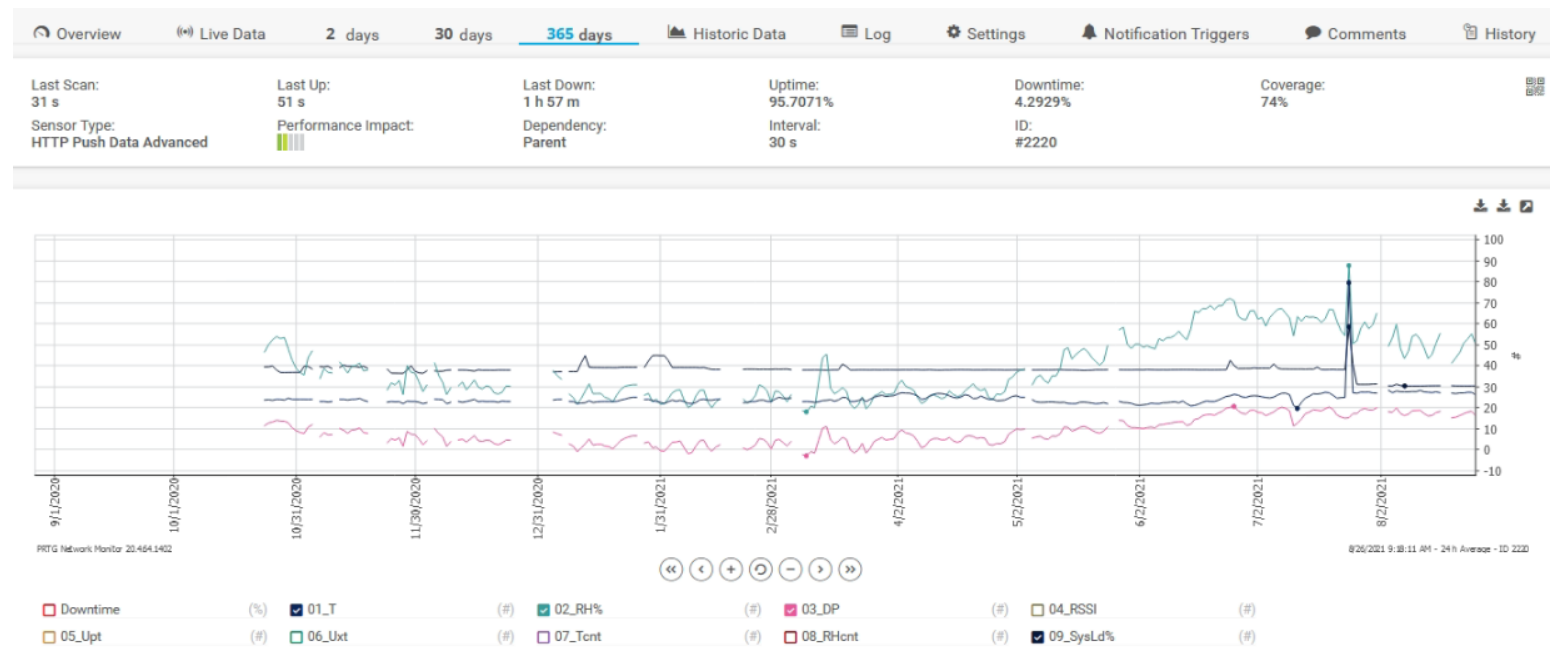

Figure 13. PRTG Server data for temperature, humidity, dew point and SysLoad

For collecting data, a free version of the mentioned software is used that can integrate 100 sensors only. In the Figure 13 is performed a 365-day filter, filter applied for sensors with temperature, relative humidity, dew point or SysLoad for the proposed architecture. The spikes from the figure are for alarms testing purpose only and to test if everything in the proposed architecture is working. In Figure 14 some data are depicted for the same data and the same filter but is added the DownTime of the system.
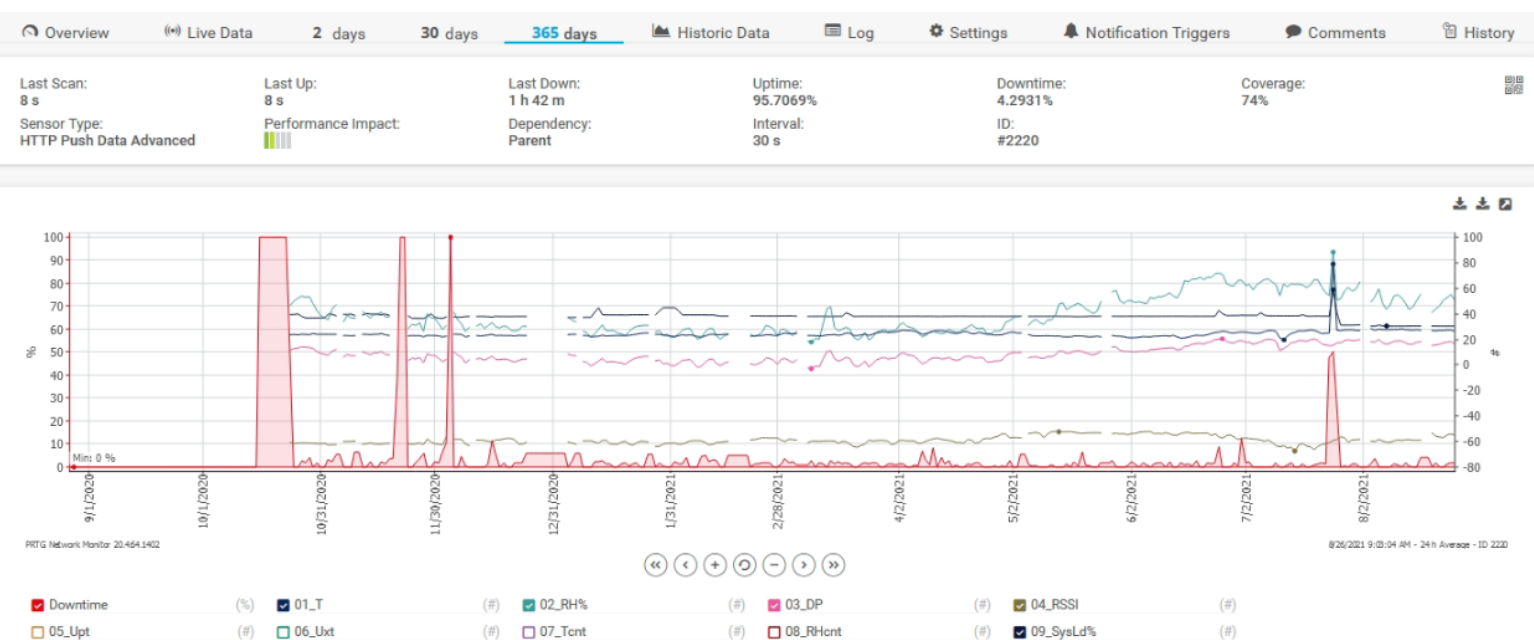

Figure 14. PRTG Server data for sensors and DownTime 


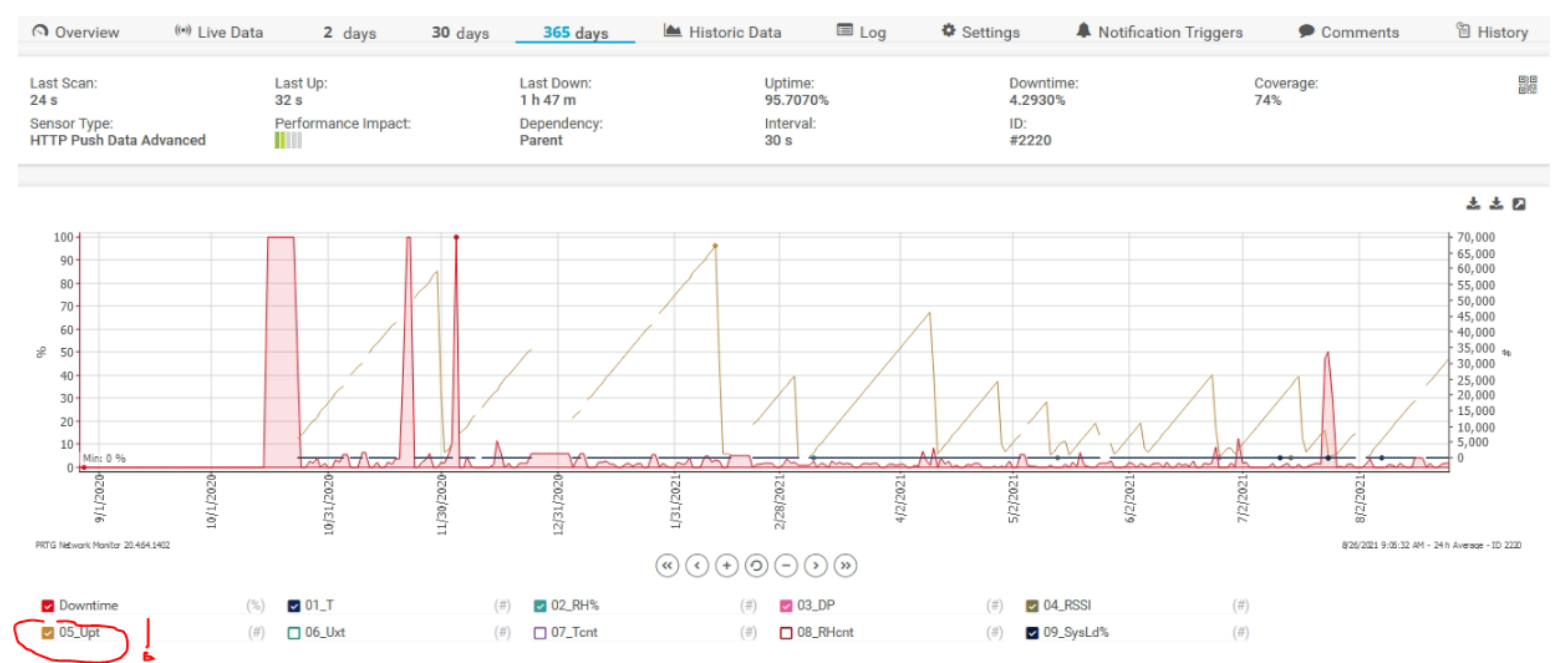

Figure 14. Microclimate monitoring in LV/MV switchgear using PRTG Server - 365 days filter, Sensor UpTime filter checked, name 05_Upt

DownTime intervals are for server time stability tests. The UpTime meter called 05_Upt was reset for each voltage failure at the sensor, and you can see on the graph how many voltage interruptions there were at the measurement sensor.

\section{Conclusions}

If a PD starts, the damages of the electrical equipment will exponentially increase and could cause safety issues, the process being irreversible.

The LV/MV switchgear cells monitoring systems are important in optimizing the maintenance costs of the Electric Grid distribution points. Using partial discharge as a health indicator in electric power assets is somewhat like listening heart rate or blood pressure monitoring in human health. While high levels can be useful to diagnose a problem, they need to be looked at in context and trended over time. While partial discharge analysis is not easy and is often confusing, it provides an early warning on asset failure, and as such, should be included in any comprehensive asset health monitoring program.

There is a tradeoff between very expensive and complicated diagnostic tools and simpler, low-cost sensor devices. The simple, low-cost devices are:

- Intended for deployment in every LV, MV and HV asset.

- Require some form of confirmation before action is taken.

- Are best used to signal a need for professional diagnostic services unless an obvious root cause is found on visual inspection.

Partial discharge alarms are informative, have the role of prediction, and should not be a distraction from main operations. Normally, these alarms should be routed to asset management teams. The life of the switchgear can often be extended by incorporating appropriate predictive and preventive maintenance.

In this paper is presented a low-cost and efficient platform for IoT monitoring the LV/MV switchgear cells. The proposed architecture is defined after the real-life problems meet at ElectroAlfa company and its current implemented to optimize the maintenance costs of the sold developed products.

Author Contributions: Conceptualization, R.F. and A.P.; methodology, R.F. and A.G.; software, R.F.; validation, R.F., A.P. and A.G.; formal analysis, R.F.; investigation, R.F.; resources, R.F., A.P. and A.G.; data curation, R.F.; writing-original draft preparation, R.F. and A.P.; writing-review and editing, A.P.; visualization, R.F.; supervision, A.G.; project administration, A.G.; funding acquisition, A.G. All authors have read and agreed to the published version of the manuscript.

Funding: This work was supported by the project "Integrated Center for Research, Development and Innovation in Advanced Materials, Nanotechnologies, and Distributed Systems for Fabrication and Control (MANSiD)" and by the project "Supporting the research of excellence within the Ștefan 
cel Mare University of Suceava" financed by the Romania National Council for Higher Education Funding, CNFIS, project number CNFIS-FDI-2020-0615.

Institutional Review Board Statement: Not applicable.

Informed Consent Statement: Not applicable.

Conflicts of Interest: The authors declare no conflict of interest.

\section{References}

1. Peng, K. Equipment Management in the Post-Maintenance Era: A New Alternative to Total Productive Maintenance (TPM). CRC Press. ISBN-9781466501942, 2012, 132-136.

2. Upton, D.W.; Mistry, K.K.; Mather, P.J.; Zaharis, Z.D.; Atkinson, R.C.; Tachtatzis, C.; Lazaridis, P.I. A Review of Techniques for RSS-Based Radiometric Partial Discharge Localization. Sensors 2021, 21, 909.

3. Nagdev, A.; Tarun, G. Fault Class Prediction in Unsupervised Learning using Model-Based Clustering Approach. 2018 International Conference on Information and Computer Technologies (ICICT), 2018, 5-12.

4. Rykov, M. The Top 10 Industrial AI use cases. Available online: https://iot-analytics.com/the-top-10-industrial-ai-use-cases (accessed on 28 July 2021).

5. MV Switchgear. Available online: https://electroalfa.ro/referinte-infrastructural, accessed on 08 September 2021)

6. Wu, M.; Cao, H.; Cao, J.; Nguyen, H.; Gomes, J. B.; Krishnaswamy, S. P. An overview of state-of-the-art partial discharge analysis techniques for condition monitoring. IEEE Electrical Insulation Magazine, 31 (6), 2015, 22-35.

7. Karimi, M.; Majidi, M.; MirSaeedi, H.; Arefi, M. M.; Oskuoee, M. A Novel Application of Deep Belief Networks in Learning Partial Discharge Patterns for Classifying Corona, Surface, and Internal Discharges. IEEE Transactions on Industrial Electronics, 67 (4), 2020, 3277-3287.

8. IEC 60270, High-voltage test techniques-Partial discharge measurement, Version 2000, 3rd ed.; IEC: Geneva, Switzerland, 2000.

9. Janus, P. Acoustic Emission Properties of Partial Discharges in the Time-Domain and Their Applications. EES Examensarbete/Master Thesis, XR-EE-ETK 2012 004, 2012, 7-12.

10. Xiao, S.; Moore, P.; Judd, M.; Portugues, I. An investigation into electromagnetic radiation due to partial discharges in high voltage equipment. Proceedings of the 2007 IEEE Power Engineering Society General Meeting, 2007, 1-7.

11. Davies, N.; Tang, J.; Shiel, P. Benefits and experiences of non-intrusive partial discharge measurements on MV Switchgear. 19th International Conference on Electricity Distribution, 2007.

12. Lemesch, G. Ozone Measurement - A Diagnosis Tool for PD-Detection in Large Generators. Iris Rotating Machine Conference, 2004.

13. Javed, H.; Li, K.; Zhang, G.-Q.; Plesca, A. T. Online Monitoring of Partial Discharge by Measuring Air Decomposition by products under Low and High Humidity. 2017 2nd International Conference on Power and Renewable Energy (ICPRE), 2017, $244-249$.

14. VanHaeren, R.; Stone, G.; Meehan, J.; Kurtz, M. Preventing failures in outdoor distribution-class metalclad switchgear. IEEE Trans. Power Appar. Syst. 1985, 2706-2712.

15. Stone, G. C.; Warren, V. Advancements in interpreting partial discharge test results to assess stator winding condition. IEEEIAS/PCA 2004 Cement Industry Technical Conference, 2004, 61-65.

16. EATechnology. Available online: www.eatechnology.com, (accessed on 05 October 2021).

17. Goldthorpe, S.; Ferris, R.; Hodgson, S.; Use of web based partial discharge monitoring to extend asset life. 20th International Conference on Electricity Distribution, 2009.

18. Banks A.; Gupta R. OASIS standard - MQTT Version 3.1.1 Available online: https://docs.oasis-open.org/mqtt/mqtt/v3.1.1/mqttv3.1.1.html, (accessed on 28 July 2021).

19. YangQun L. An Integrated Platform for the Internet of Things Based on an Open-Source Ecosystem, Future Internet, 10 (11), 2018, 105. 